\title{
EXTREMAL PROBLEMS IN THE CLASS OF CLOSE-TO-CONVEX FUNCTIONS $\left.{ }^{(}\right)$
}

\author{
BY \\ BERNARD PINCHUK( $\left.{ }^{2}\right)$
}

\begin{abstract}
The class $K$ of normalized close-to-convex functions in $D=\{z:|z|<1\}$ has a parametric representation involving two Stieltjes integrals. Using a variational method due to G. M. Goluzin [2] for classes of analytic functions defined by a Stieltjes integral, variational formulas are developed for $K$. With these variational formulas, two general extremal problems within $K$ are solved.

The first problem is to maximize the functional $\operatorname{Re} F\left[\log f^{\prime}(z)\right]$ over $K$ where $F(w)$ is a given entire function anu $z$ a given point in $D$. A special case of this is the rotation theorem for $K$. The second problem solved is a general coefficient problem. Both problems are solved by characterizing the measures which appear in the integral representation for the extremal functions.

The classes of convex univalent functions in $D$ and functions whose derivative has a positive real part in $D$ are proper subclasses of $K$. The methods used to solve the extremal problems in $K$ can be used for these subclasses as well. Some of the results for the subclasses are known and are not presented here, even though the methods differ from those used previously.

It should be mentioned that Goluzin originally used these methods to solve extremal problems of the first type mentioned above within the classes of starlike and typically real functions.
\end{abstract}

\section{Preliminaries.}

Definition. A function $f(z)$ analytic in $D$, is said to be close-to-convex in $D$ if there exists a convex univalent function $\phi(z)$ such that

$$
\operatorname{Re} \frac{f^{\prime}(z)}{\phi^{\prime}(z)}>0
$$

for all $z \in D$.

Without loss of generality we assume that $f(z)$ is normalized, i.e., $f(0)=0$ and $f^{\prime}(0)=1$, and furthermore that $\phi(0)=0$ and $\left|\phi^{\prime}(0)\right|=1$. We denote this class of normalized close-to-convex functions by $K$. W. Kaplan [5], who first introduced

Received by the editors September 14, 1966.

( $\left.{ }^{1}\right)$ This research forms part of the author's doctoral dissertation at the Belfer Graduate School of Science of Yeshiva University, written under the direction of Professor Harry E. Rauch.

( $\left.{ }^{2}\right)$ Research partially sponsored by the Air Force Office of Scientific Research, Office of Aerospace Research, U.S. Air Force, under AFOSR Grant No. AF-AFOSR 1077-66. 
close-to-convex functions, proved that every close-to-convex function is univalent. Thus $K$ is a subclass of $S$, the class of normalized univalent functions in $D$.

Let $C$ denote the class of normalized convex functions in $D$, i.e., the subclass of $S$ consisting of those functions which map $D$ onto a convex domain. Let $\mathscr{P}^{\prime}$ denote the class of normalized analytic functions whose derivative has a positive real part in $D . \mathscr{P}^{\prime}$ is a subclass of $S$ and furthermore, both $\mathscr{P}^{\prime}$ and $C$ are contained in $K$.

The classes $C$ and $\mathscr{P}^{\prime}$ can be defined analytically by the following well-known (see e.g., [6, p. 224]) conditions:

LEMMA 1.1. Let $f(z)=z+a_{2} z^{2}+\cdots$ be analytic in $D$. Then $f(z) \in C$ if and only if

$$
\operatorname{Re}\left\{1+\frac{z f^{\prime \prime}(z)}{f^{\prime}(z)}\right\}>0
$$

for all $z \in D$.

The definition of $\mathscr{P}^{\prime}$ can be formulated as follows:

Under the hypothesis of Lemma $1.1, f(z) \in \mathscr{P}^{\prime}$ if and only if

$$
\operatorname{Re} f^{\prime}(z)>0
$$

for all $z \in D$.

Using the integral representation for analytic functions with positive real part in $D$ (Herglotz's Theorem, see e.g., [3, p. 68]) together with (1.2) and (1.3) we obtain the following well-known integral representations for $C$ and $\mathscr{P}^{\prime}$.

Let $I$ denote the class of nondecreasing functions $\alpha(t)$ in the interval $-\pi \leqq t \leqq \pi$ which satisfy

$$
\int_{-\pi}^{\pi} d \alpha(t)=\alpha(\pi)-\alpha(-\pi)=1
$$

LEMMA 1.2. (a) $f(z) \in C$ if and only if

$$
f^{\prime}(z)=\exp \left(\int_{-\pi}^{\pi}-2 \log \left(1-z e^{-i t}\right) d \alpha(t)\right)
$$

for some $\alpha(t) \in I$.

(b) $f(z) \in \mathscr{P}^{\prime}$ if and only if

$$
f^{\prime}(z)=\int_{-\pi}^{\pi} \frac{e^{i t}+z}{e^{i t}-z} d \beta(t)
$$

for some $\beta(t) \in I$.

Using Herglotz's theorem together with (1.1) and (1.4) we obtain the integral representation for the class $K$.

Let $f(z)=z+a_{2} z^{2}+\cdots \in K$ with associated convex function $\phi(z)=e^{i \gamma} z+b_{2} z^{2}+\cdots$. Then

$$
\frac{f^{\prime}(z)}{\phi^{\prime}(z)}=P(z)=e^{-i \gamma}+P_{1} z+\cdots,
$$


where

$$
\operatorname{Re} P(z)>0
$$

for $z \in D$, and $e^{-i \gamma} \phi(z) \in C$.

By Herglotz's theorem, (1.6) is equivalent to

$$
P(z)=\cos \gamma \int_{-\pi}^{\pi} \frac{e^{i t}+z}{e^{i t}-z} d \alpha(t)-i \sin \gamma
$$

for some $\alpha(t) \in I$ and for $|\gamma|<\pi / 2$.

We thus have

LEMMA 1.3. $f(z) \in K$ if and only if

$$
\begin{aligned}
f^{\prime}(z)= & e^{i \gamma}\left(\cos \gamma \int_{-\pi}^{\pi} \frac{e^{i t}+z}{e^{i t}-z} d \alpha(t)-i \sin \gamma\right) \\
& \times \exp \left(\int_{-\pi}^{\pi}-2 \log \left(1-z e^{-i t}\right) d \beta(t)\right)
\end{aligned}
$$

for some $\alpha(t)$ and $\beta(t)$, both in $I$, and $|\gamma|<\pi / 2$.

2. Variational formulas. Let Eg denote the class of analytic functions defined by a Stieltjes integral

$$
f(z)=\int_{-\pi}^{\pi} g(z, t) d \alpha(t),
$$

where $g(z, t)$ is a given function, regular in $D$ for $-\pi \leqq t \leqq \pi$ and $\alpha(t) \in I$. G. M. Goluzin [2] gave two types of variations for functions in $\mathrm{Eg}$, each obtained by a suitable variation of the function $\alpha(t)$.

We state these variational formulas and omit the proofs which can be found in [2].

I. Let $f(z)=\int_{-\pi}^{\pi} g(z, t) d \alpha(t)$ be a function in Eg.

Let $t_{1}, t_{2}$ with $t_{1}<t_{2}$ be arbitrary numbers in the interval $-\pi \leqq t \leqq \pi$, let $\lambda$ vary in the interval $-1 \leqq \lambda \leqq 1$. There exists a real number $C$, depending on $f(z), t_{1}$ and $t_{2}$, but not on $\lambda$ and $t$ such that the functions

$$
f_{*}(z)=f(z)+\lambda \int_{t_{1}}^{t_{2}} \frac{\partial g(z, t)}{\partial t}|\alpha(t)-C| d t
$$

are also in Eg.

Equation (2.1) is the first variational formula for Eg.

II. Let $f(z)=\int_{-\pi}^{\pi} g(z, t) d \alpha(t)$ be a function in Eg. Let $\tau_{1}, \tau_{2}$ with $-\pi \leqq \tau_{1}<\tau_{2} \leqq \pi$ be jump points for the function $\alpha(t)$, and let $\eta$ vary in the interval $-\delta \leqq \eta \leqq \delta$ where $\delta$ is determined by $f(z), \tau_{1}$ and $\tau_{2}$. Then the functions

$$
f_{* *}(z)=f(z)+\eta\left(g\left(z, \tau_{1}\right)-g\left(z, \tau_{2}\right)\right)
$$

are also functions in $\mathrm{Eg}$. 
Equation (2.2) is the second variational formula for Eg.

Variational formulas for $\mathscr{P}^{\prime}, C$ and $K$ are obtained by applying (2.1) and (2.2) to the integral representations of the previous section.

In what follows, $t_{1}, t_{2}, \tau_{1}, \tau_{2}, C, \lambda$, and $\eta$ have the same meaning as above. It is more convenient to have variational formulas for the derivatives of functions in the classes under consideration.

Variations for $\mathscr{P}^{\prime}$. The derivatives of functions in $\mathscr{P}^{\prime}$ form a class $\mathrm{Eg}$ with $g(z, t)=\left(1+z e^{-i t}\right)\left(1-z e^{-i t}\right)^{-1}$. The variational formulas for $P^{\prime}$ are

$$
f_{*}^{\prime}(z)=f^{\prime}(z)+\lambda \int_{t_{1}}^{t_{2}} \frac{2 i z e^{i t}}{\left(e^{i t}-z\right)^{2}}|\alpha(t)-C| d t
$$

and

$$
f_{* *}^{\prime}(z)=f^{\prime}(z)+\eta\left(\frac{z}{e^{i \tau_{1}}-z}-\frac{z}{e^{i \tau_{2}}-z}\right)
$$

Variations for $C$. The integral representation (1.4) can be written as

$$
f^{\prime}(z)=\exp \left(\int_{-\pi}^{\pi}-2 \log \left(1-z e^{-i t}\right) d \alpha(t)\right)=\exp G(z) .
$$

The functions $G(z)$ form a class $\mathrm{Eg}$ with $g(z, t)=-2 \log \left(1-z e^{-i t}\right)$. Variations within $C$ are obtained by varying within this $\mathrm{Eg}$. We have

$$
f_{*}^{\prime}(z)=\exp \left(G_{*}(z)\right)=\exp \left(G(z)+\lambda \int_{t_{1}}^{t_{2}} \frac{2 i z e^{-i t}}{1-z e^{-i t}}|\alpha(t)-C| d t\right) .
$$

For small values of $\lambda$ we have

$$
f_{*}^{\prime}(z)=f^{\prime}(z)+\lambda \int_{t_{1}}^{t_{2}} f^{\prime}(z) \frac{i z e^{-i t}}{1-z e^{-i t}}|\alpha(t)-C| d t+O\left(\lambda^{2}\right) .
$$

Similarly, the second variational formula for $C$ is

$$
f_{* *}^{\prime}(z)=f^{\prime}(z)+\eta f^{\prime}(z)\left(\log \left(1-z e^{-i \tau_{1}}\right)-\log \left(1-z e^{-i \tau_{2}}\right)\right)+O\left(\eta^{2}\right) .
$$

Variations for $K$. The integral representation (1.7) for $K$ can be written as

$$
f^{\prime}(z)=P_{0}(z) \phi_{0}^{\prime}(z)=(\cos \gamma P(z)-i \sin \gamma) e^{i \gamma} \phi^{\prime}(z),
$$

where

$$
P(z)=\int_{-\pi}^{\pi} \frac{e^{i t}+z}{e^{i t}-z} d \beta(t)
$$

and

$$
\phi^{\prime}(z)=\exp \left(\int_{-\pi}^{\pi}-2 \log \left(1-z e^{-i t}\right) d \alpha(t)\right),
$$

with $\alpha(t)$ and $\beta(t)$ in $I$.

Variational formulas for $K$ are obtained by varying $P(z)$ and $\phi(z)$ independently. 
From (2.3), we have

$$
\begin{aligned}
f_{*, a}^{\prime}(z) & =\left(\cos \gamma P_{*}(z)-i \sin \gamma\right) \phi_{0}^{\prime}(z) \\
& =f^{\prime}(z)+\lambda \int_{t_{1}}^{t_{2}} f^{\prime}(z) \frac{\cos \gamma 2 i z e^{i t}}{P_{0}(z)\left(e^{i t}-z\right)^{2}}|\beta(t)-C| d t .
\end{aligned}
$$

From (2.5) we have

$$
\begin{aligned}
f_{*, b}^{\prime}(z) & =P_{0}(z) e^{i \gamma} \phi_{*}^{\prime}(z) \\
& =f^{\prime}(z)+\lambda \int_{t_{1}}^{t_{2}} f^{\prime}(z) \frac{2 i z e^{-i t}}{1-z e^{-i t}}|\alpha(t)-C| d t+O\left(\lambda^{2}\right) .
\end{aligned}
$$

In the case where $\beta(t)$ or $\alpha(t)$ have jump points we have

$$
\begin{aligned}
f_{* *, a}^{\prime}(z) & =\left(\cos \gamma P_{* *}(z)-i \sin \gamma\right) \phi_{0}^{\prime}(z) \\
& =f^{\prime}(z)+\eta\left(\frac{z \cos \gamma f^{\prime}(z)}{P_{0}(z)\left(e^{i \tau_{1}}-z\right)}-\frac{z \cos \gamma f^{\prime}(z)}{P_{0}(z)\left(e^{i \tau_{2}}-z\right)}\right)
\end{aligned}
$$

and

$$
\begin{aligned}
f_{* *, b}^{\prime}(z) & =P_{0}(z) e^{i \gamma} \phi_{* *}^{\prime}(z) \\
& =f^{\prime}(z)+\eta f^{\prime}(z)\left(\log \left(1-z e^{i \tau_{1}}\right)-\log \left(1-z e^{-i \tau_{2}}\right)\right)+O\left(\eta^{2}\right) .
\end{aligned}
$$

We now state a lemma, the proof of which is obvious.

LEMMA 2.1. Let $Q(t)$ be a continuous function having at most $N$ zeros in the interval $-\pi<t \leqq \pi$, and let $\alpha(t) \in I$. To each interval $\left(t_{i}, t_{j}\right)$ where $-\pi \leqq t_{i}<t_{j} \leqq \pi$ let there be associated a constant $C_{i j}$. Then, the condition

$$
\int_{t_{i}}^{t_{j}} Q(t)\left|\alpha(t)-C_{i j}\right| d t=0
$$

for all intervals $\left(t_{i}, t_{j}\right)$ implies that $\alpha(t)$ is a step function having at most $N$ jump points. Furthermore, the jump points can only be at the zeros of $Q(t)$.

3. An extremal problem for $K$. The variational formulas of the preceding section can be used to solve extremal problems within the various classes. We first consider a general extremal problem which contains, as a special case, the "rotation" and "distortion" theorems. Goluzin [2] solved this problem for the class of starlike functions.

THEOREM 3.1. For a given entire function $F(w)$ and a given point $z \in D$, the maximum for either of the functionals

$$
L\left(f^{\prime}\right)=\operatorname{Re} F\left[\log f^{\prime}(z)\right], \quad l\left(f^{\prime}\right)=\left|F\left[\log f^{\prime}(z)\right]\right|
$$

in the class $K$ is attained only for a function of the form

$$
f^{\prime}(z)=\left(1-z e^{i \alpha}\right)^{-2}\left(1+z e^{i \delta}\right)\left(1-z e^{i \beta}\right)^{-1}
$$

where $\alpha, \beta$, and $\delta$ are in the interval $[-\pi, \pi]$. 
Hence, for $z \in D$ we have the following bounds

$$
\begin{gathered}
\operatorname{Re} F\left[\log f^{\prime}(z)\right] \leqq \operatorname{Max}_{\alpha, \beta, \delta} \operatorname{Re}\left\{F\left[\log \left(1-z e^{i \alpha}\right)^{-2}\left(1+z e^{i \delta}\right)\left(1-z e^{i \beta}\right)^{-1}\right]\right\}, \\
\left|F\left[\log f^{\prime}(z)\right]\right| \leqq \operatorname{Max}_{\alpha, \beta, \delta}\left|F\left[\log \left(1-z e^{i \alpha}\right)^{-2}\left(1+z e^{i \delta}\right)\left(1-z e^{i \beta}\right)^{-1}\right]\right| .
\end{gathered}
$$

Proof. The compactness of $K$ assures the existence of an extremal function for the problems under consideration. Furthermore, a function which maximizes $l\left(f^{\prime}\right)$ will simultaneously maximize $\operatorname{Re}\left\{e^{i \gamma} F\left[\log f^{\prime}(z)\right]\right\}$ with suitably chosen $\gamma$. Hence it is sufficient to prove the theorem for the functional $L\left(f^{\prime}\right)$.

Let $f(z)$ be an extremal function with

$$
\begin{aligned}
f^{\prime}(z)= & \left(\cos \gamma \int_{-\pi}^{\pi} \frac{e^{i t}+z}{e^{i t}-z} d \beta(t)-i \sin \gamma\right) e^{i \gamma} \\
& \times \exp \left(\int_{-\pi}^{\pi}-2 \log \left(1-z e^{-i t}\right) d \alpha(t)\right) .
\end{aligned}
$$

We compute $L\left(f^{\prime}\right)$ for the varied functions $f_{*, a}^{\prime}(z)$ and $f_{*, b}^{\prime}(z)((2.7)$ and $(2.8)$ respectively). Since

$$
F\left[\log f_{*, a}^{\prime}(z)\right]=F\left[\log f^{\prime}(z)\right]+\lambda \int_{t_{1}}^{t_{2}} F^{\prime}\left[\log f^{\prime}(z)\right] \frac{\cos \gamma 2 i z e^{i t}}{P_{0}(z)\left(e^{i t}-z\right)^{2}}|\beta(t)-C| d t+O\left(\lambda^{2}\right)
$$

we have

$$
L\left(f_{*, a}^{\prime}\right)=L\left(f^{\prime}\right)+\lambda \int_{t_{1}}^{t_{2}} \operatorname{Re}\left\{F^{\prime}\left[\log f^{\prime}(z)\right] \frac{\cos \gamma 2 i z e^{i t}}{P_{0}(z)\left(e^{i t}-z\right)^{2}}\right\}|\beta(t)-C| d t+O\left(\lambda^{2}\right)
$$

Similarly, since

$$
F\left[\log f_{*, b}^{\prime}(z)\right]=F\left[\log f^{\prime}(z)\right]+\lambda \int_{t_{1}}^{t_{2}} F^{\prime}\left[\log f^{\prime}(z)\right] \frac{2 i z e^{-i t}}{1-z e^{-i t}}|\alpha(t)-C| d t+O\left(\lambda^{2}\right),
$$

we have

$$
L\left(f_{*, b}^{\prime}\right)=L\left(f^{\prime}\right)+\lambda \int_{t_{1}}^{t_{2}} \operatorname{Re}\left\{F^{\prime}\left[\log f^{\prime}(z)\right] \frac{2 i z e^{-i t}}{1-z e^{-i t}}\right\}|\alpha(t)-C| d t+O\left(\lambda^{2}\right)
$$

The extremal property of the function $f(z)$ implies

$$
L\left(f_{*, a}^{\prime}\right) \leqq L\left(f^{\prime}\right) \text { and } L\left(f_{*, b}^{\prime}\right) \leqq L\left(f^{\prime}\right) .
$$

We thus conclude that a necessary condition for $f(z)$ to be an extremal function is that the coefficient of $\lambda$ in (3.2) and (3.3) should vanish, i.e.,

$$
\int_{t_{1}}^{t_{2}} \operatorname{Re}\left\{F^{\prime}\left[\log f^{\prime}(z)\right] \frac{i z e^{i t}}{P_{0}(z)\left(e^{i t}-z\right)^{2}}\right\}|\beta(t)-C| d t=0
$$

and

$$
\int_{t_{1}}^{t_{2}} \operatorname{Re}\left\{F^{\prime}\left[\log f^{\prime}(z)\right] \frac{i z e^{-i t}}{i-z e^{-i t}}\right\}|\alpha(t)-C| d t=0
$$


If we set

$$
Q_{1}(t)=\operatorname{Re}\left\{F^{\prime}\left[\log f^{\prime}(z)\right] \frac{i z e^{i t}}{P_{0}(z)\left(e^{i t}-z\right)^{2}}\right\}
$$

the equation $Q_{1}(t)=0$ is equivalent to

$$
\operatorname{Re}\left\{F^{\prime}\left[\log f^{\prime}(z)\right] i z \overline{P_{0}(z)}\left(\bar{z}^{2} e^{i t}-2 \bar{z}+e^{-i t}\right)\right\}=0
$$

i.e., a quadratic equation in $e^{i t}$. Hence, $Q_{1}(t)$ has at most two zeros for $-\pi \leqq t<\pi$. Similarly, if we set

$$
Q_{2}(t)=\operatorname{Re}\left\{F^{\prime}\left[\log f^{\prime}(z) \frac{i z e^{-i t}}{1-z e^{-i t}}\right\}\right.
$$

the equation $Q_{2}(t)=0$ is equivalent to

$$
\operatorname{Re}\left\{F^{\prime}\left[\log f^{\prime}(z)\right] i e^{-i t} z\left(1-\bar{z} e^{i t}\right)\right\}=0,
$$

i.e., a quadratic equation in $e^{i t}$ and hence $Q_{2}(t)$ has at most two zeros for $-\pi \leqq t<\pi$. By a recent theorem of Kirwan [9], we know that $F^{\prime}\left[\log f^{\prime}(z)\right] \neq 0$.

Using Lemma 2.1, we conclude that $\alpha(t)$ and $\beta(t)$ are step functions, each having at most two jump points in $-\pi \leqq t<\pi$. Furthermore, the jump points of $\beta(t)$ must be zeros of $Q_{1}(t)$ and the jump points of $\alpha(t)$ must be zeros of $Q_{2}(t)$.

We now assume that $\alpha(t)$ has two jump points which we call $t_{1}$ and $t_{2}$ and that $\beta(t)$ has two jump points which we call $t_{3}$ and $t_{4}$. The variation functions $f_{* *, a}^{\prime}(z)$ and $f_{* *, b}^{\prime}(z)$ can now be constructed. From (2.9) and (2.10) we have

$$
L\left(f_{* *, a}^{\prime}\right)=L\left(f^{\prime}\right)+\eta \operatorname{Re}\left\{F^{\prime}\left[\log f^{\prime}(z)\right]\left(z / P_{0}(z)\left(e^{i t_{3}}-z\right)-z / P_{0}(z)\left(e^{i t_{4}}-z\right)\right)\right\}+O\left(\eta^{2}\right)
$$

and

$$
L\left(f_{* *, b}^{\prime}\right)=L\left(f^{\prime}\right)+\eta \operatorname{Re}\left\{F^{\prime}\left[\log f^{\prime}(z)\right]\left(\log \left(1-z e^{-i t_{1}}\right)-\log \left(1-z e^{-i t_{2}}\right)\right)+O\left(\eta^{2}\right) .\right.
$$

The extremal property then leads to the condition

$$
\operatorname{Re}\left\{F^{\prime}\left[\log f^{\prime}(z)\right]\left(z / P_{0}(z)\left(e^{i t_{3}}-z\right)-z / P_{0}(z)\left(e^{i t_{4}}-z\right)\right)\right\}=0
$$

and

$$
\operatorname{Re}\left\{F^{\prime}\left[\log f^{\prime}(z)\right]\left(\log \left(1-z e^{-i t_{1}}\right)-\log \left(1-z e^{-i t_{2}}\right)\right)\right\}=0 .
$$

Condition (3.6) implies that the function

$$
\operatorname{Re}\left\{F^{\prime}\left[\log f^{\prime}(z)\right] z / P_{0}(z)\left(e^{i t}-z\right)\right\}
$$

has the same value for $t=t_{3}$ and $t=t_{4}$. Thus, the derivative of this function with respect to $t$ must vanish at a point $t$ in the interval $\left(t_{3}, t_{4}\right)$. However, this implies that $Q_{1}(t)$ will vanish at three points in $-\pi \leqq t<\pi$ which is impossible. Hence, $\beta(t)$ can only have a single jump point.

Similarly, we conclude from condition (3.7) that $\alpha(t)$ can have only a single jump point. 
Evaluating (1.7) where $\alpha(t)$ and $\beta(t)$ are step functions with a single jump each, we see that the function must have the required form.

Application. Setting $F(w)= \pm i w$ in $L\left(f^{\prime}\right)$ of Theorem 3.1, we obtain the rotation theorem for $K\left(^{3}\right)$.

COROLlaRY 3.1. The functional $\left|\arg f^{\prime}(z)\right|$ attains its maximum in the class $K$ only for a function of the form

$$
f^{\prime}(z)=\left(1-z e^{i \alpha}\right)^{-2}\left(1-z e^{i \delta}\right)\left(1-z e^{i \beta}\right)^{-1}
$$

where $\alpha, \beta$, and $\delta$ are in the interval $[-\pi, \pi]$.

Thus, for $z \in D$,

$$
\begin{aligned}
\left|\arg f^{\prime}(z)\right| & \leqq \operatorname{Max}_{\alpha, \beta, \delta}\left|-2 \arg \left(1-z e^{i \alpha}\right)+\arg \left(1-z e^{i \delta}\right)-\arg \left(1-z e^{i \beta}\right)\right| \\
& =4 \sin ^{-1}|z|
\end{aligned}
$$

where $\sin ^{-1} 0=0$.

It has been pointed out by Professor D. J. Newman that the inequality

$$
\left|\arg f^{\prime}(z)\right| \leqq 4 \sin ^{-1}(z)
$$

follows from the integral representation (1.7). This is because the integrals appearing in (1.7) are convex combinations of the integrands and therefore it suffices to have bounds on the arguments of the integrands. Of course, this does not give the form of the extremal function.

A rotation theorem is known for a slightly more restricted class than $K$ (see [7]).

4. The class $\mathscr{P}^{\prime}$. The variational formulas (2.3) and (2.4) for the class $\mathscr{P}^{\prime}$ are identical to the formulas (2.7) and (2.9) for the class $K$ except for the factor $\left(f^{\prime}(z) \cos \gamma\right) / P_{0}(z)$ which appears in (2.7) and (2.9), and that the $f(z)$ appearing in (2.3) and (2.4) is a function in $\mathscr{P}^{\prime}$ and the $f(z)$ appearing in (2.7) and (2.9) is a function in $K$. These modifications do not affect the argument given in $\$ 3$ to prove that $\beta(t)$ is a step function having a single jump point. We therefore have the following theorem for $\mathscr{P}^{\prime}$.

THEOREM 4.1. For a given entire function $F(w)$ and a given point $z \in D$, the maximum for either of the functionals

$$
L\left(f^{\prime}\right)=\operatorname{Re} F\left[\log f^{\prime}(z)\right], l\left(f^{\prime}\right)=\left|F\left[\log f^{\prime}(z)\right]\right|
$$

in the class $\mathscr{P}^{\prime}$ is attained only for a function of the form

$$
f^{\prime}(z)=\left(1+z e^{i \alpha}\right)\left(1-z e^{i \alpha}\right)^{-1}, \quad-\pi \leqq \alpha \leqq \pi .
$$

( ${ }^{3}$ I have recently been informed by Professor James Hummel that this bound on $\left|\arg f^{\prime}(z)\right|$ is known. See [10]. 
Hence, for $z \in D$ we have the following bounds

$$
\begin{gathered}
\operatorname{Re} F\left[\log f^{\prime}(z)\right] \leqq \operatorname{Max}_{\alpha} \operatorname{Re} F\left[\log \left(1+z \varepsilon^{i \alpha}\right)-\log \left(1-z \varepsilon^{i \alpha}\right)\right], \\
\left|F\left[\log f^{\prime}(z)\right]\right| \leqq \operatorname{Max}_{\alpha} \mid F\left[\log \left(1+z e^{i \alpha}\right)-\log \left(1-z e^{i \alpha}\right) \mid .\right.
\end{gathered}
$$

Proof. The compactness of $\mathscr{P}^{\prime}$ assures the existence of a function in $\mathscr{P}^{\prime}$ which is extremal for the problems under consideration. Furthermore, it suffices to prove the theorem for the functional $L(f)$.

Let $f(z)$ be an extremal function with

$$
f^{\prime}(z)=\int_{-\pi}^{\pi} \frac{1+z e^{-i t}}{1-z e^{-i t}} d \alpha(t) .
$$

Computing $L\left(f_{*}^{\prime}\right)$ for the varied function (2.3), we apply the arguments of the previous section to prove that $\alpha(t)$ is a step function having a single jump point, say at $t=-\alpha$. Evaluating (4.1) with such an $\alpha(t)$ we have the desired result.

COROLlaRY 4.1. The functional $\left|\arg f^{\prime}(z)\right|$ attains its maximum in the class $\mathscr{P}^{\prime}$ only for a function of the form

Thus, for $z \in D$

$$
f^{\prime}(z)=\left(1+z e^{i \alpha}\right)\left(1-z e^{i \alpha}\right)^{-1}, \quad-\pi \leqq \alpha \leqq \pi .
$$

$$
\left|\arg f^{\prime}(z)\right| \leqq \operatorname{Max}_{\alpha}\left|\arg \left(1+z e^{i \alpha}\right)\left(1-z e^{i \alpha}\right)^{-1}\right|=\sin ^{-1}\left(2|z| / 1+|z|^{2}\right)
$$

where $\sin ^{-1} 0=0$.

This corollary is obtained by setting $F(w)= \pm i w$ in $L(f)$ of Theorem 4.1. The observation of Professor D. J. Newman mentioned in $\S 3$ applies here as well.

5. The class $C$. The variational formulas (2.5) and (2.6) for $C$ are identical to formulas (2.8) and (2.10) for $K$ except that the $f^{\prime}(z)$ appearing in (2.5) and (2.6) is a convex function and the $f^{\prime}(z)$ appearing in (2.8) and (2.10) is in $K$. This does not affect the argument given in $\S 3$ to prove that $\alpha(t)$ is a step function having a single jump. We therefore have the following theorem for $C$.

THEOREM 5.1. For a given entire function $F(w)$ and a given point $z \in D$ the maximum for either of the functionals

$$
L\left(f^{\prime}\right)=\operatorname{Re} F\left[\log f^{\prime}(z)\right], \quad l\left(f^{\prime}\right)=\left|F\left[\log f^{\prime}(z)\right]\right|
$$

in the class $C$ is attained only for a function of the form

$$
f^{\prime}(z)=\left(1-z e^{i \alpha}\right)^{-2}, \quad-\pi \leqq \alpha \leqq \pi .
$$

Thus, for $z \in D$ we have the following bounds

$$
\begin{gathered}
\operatorname{Re} F\left[\log f^{\prime}(z)\right] \leqq \operatorname{Max}_{\alpha} \operatorname{Re} F\left[\log \left(1-z e^{i \alpha}\right)^{-2}\right] \\
\left|F\left[\log f^{\prime}(z)\right]\right| \leqq \operatorname{Max}_{\alpha}\left|F\left[\log \left(1-z e^{i \alpha}\right)^{-2}\right]\right| .
\end{gathered}
$$


Proof. The compactness of $C$ assures the existence of an extremal function and furthermore, it suffices to prove the theorem for the functional $L(f)$. Let $f(z)$, with

$$
f^{\prime}(z)=\exp \left(\int_{-\pi}^{\pi}-2 \log \left(1-z e^{-i t}\right) d \alpha(t)\right),
$$

be an extremal function. Computing $L\left(f_{*}^{\prime}\right)$ for the variation function (2.5), we apply the argument given in $\S 3$ to prove that $\alpha(t)$ is a step function having a single jump point. Evaluating (5.1) gives the desired result.

Setting $F(w)= \pm i w$ in $L(f)$ of Theorem 5.1, we have the well-known rotation theorem for $C$ (see [1]).

COROLlary 5.1. The functional $\left|\arg f^{\prime}(z)\right|$ attains its maximum in the class $C$ only for a function of the form

$$
f^{\prime}(z)=\left(1-z e^{i \alpha}\right)^{-2}, \quad-\pi \leqq \alpha \leqq \pi .
$$

Thus, for $z \in D$

where $\sin ^{-1} 0=0$.

$$
\left|\arg f^{\prime}(z)\right| \leqq \operatorname{Max}_{\alpha^{\prime}}\left|\arg \left(1-z e^{i \alpha}\right)^{-2}\right|=2 \sin ^{-1}|z|
$$

Here too, the inequality $\left|\arg f^{\prime}(z)\right| \leqq 2 \sin ^{-1}|z|$ could have been derived from the representation (1.4).

6. A coefficient problem. Using methods similar to those presented in $\S 3$, we now solve a general coefficient problem for $K$. The problem can be stated as follows.

Let $F\left(X_{2}, \ldots, X_{n}\right)$ be any analytic function in $C^{n-1}$. Consider the functional $\Phi(f)=\operatorname{Re} F\left(a_{2}, \ldots, a_{n}\right)$ where $f(z)=z+\sum_{n=2}^{\infty} a_{n} z^{n}$. Maximize $\Phi(f)$ over $K$.

J. A. Hummel [4] maximized $\Phi(f)$ over the class $S^{*}$ of starlike univalent functions using the method of interior variations. The solution of the problem for $C$ then follows from the relation $f(z) \in S^{*}$ if and only if $f(z)=z g^{\prime}(z) g(z) \in C$. Hummel found a suitable interior variation which preserved starlikeness. The difficulty in using this method for the study of $K$ is that a suitable interior variation which preserves close-to-convexity is not known. M. S. Robertson [8] maximized $\Phi(f)$ over the class $\mathscr{P}$ of analytic functions which have a positive real part in $D$. The result for $\mathscr{P}^{\prime}$ follows directly from this.

We solve the extremal problem for $K$, but because of the identical variational formulas as pointed out previously, we actually have maximized $\Phi(f)$ over $S^{*}$, $C, \mathscr{P}^{\prime}$ and $\mathscr{P}$.

THeOREM 6.1. For a given function $F\left(X_{2}, \ldots, X_{n}\right)$ which is analytic in $C^{n-1}$, the functional $\Phi(f)=\operatorname{Re} F\left(a_{2}, \ldots, a_{n}\right)$ attains its maximum in the class $K$ only for a functional of the form

$$
f(z)=\int_{0}^{z} \prod_{j=1}^{N}\left(1-w e^{i \alpha j}\right)^{-2 u_{j}} \sum_{k=1}^{M} \eta_{k}\left(1+w e^{i \delta_{k}}\right)\left(1-w e^{i \beta_{k}}\right)^{-2} d w
$$


where $\quad N \leqq n-1, \quad M \leqq n-1, \quad u_{j} \geqq 0, \quad \eta_{k} \geqq 0, \quad \sum_{j=1}^{N} u_{j}=\sum_{k=1}^{M} \eta_{k}=1, \quad-\pi \leqq \alpha_{j} \leqq \pi$, $-\pi \leqq \beta_{k} \leqq \pi,-\pi \leqq \delta_{k} \leqq \pi$.

Proof. The compactness of $K$ assures the existence of a function in $K$ which maximizes $\Phi(f)$.

Let $f(z)$ be an extremal function with

$$
\begin{aligned}
f(z)= & \int_{0}^{z}\left(\cos \gamma \int_{-\pi}^{\pi} \frac{e^{i t}+w}{e^{i t}-w} d \beta(t)-i \sin \gamma\right) e^{i \gamma} \\
& \times \exp \left(\int_{-\pi}^{\pi}-2 \log \left(1-w e^{-i t}\right) d \alpha(t)\right) d w=z+\sum_{n=2}^{\infty} a_{n} z^{n} .
\end{aligned}
$$

We compute $\Phi\left(f_{*, a}\right)$ and $\Phi\left(f_{*, b}\right)$ for the variational functions (2.7) and (2.9) (which we have integrated). We have

$$
\begin{aligned}
f_{*, a}(z) & =f(z)+\int_{0}^{z} \lambda \int_{t_{1}}^{t_{2}} f^{\prime}(w) \frac{\cos \gamma 2 i w e^{i t}}{P_{0}(w)\left(e^{i t}-w\right)^{2}}|\beta(t)-C| d t d w \\
& =z+\sum_{n=2}^{\infty}\left(a_{n}+\lambda \int_{t_{1}}^{t_{2}} \delta a_{n}|\beta(t)-C| d t\right) z^{n}
\end{aligned}
$$

and

$$
\begin{aligned}
f_{*, b}(z) & =f(z)-\int_{0}^{z}\left(\lambda \int_{t_{1}}^{t_{2}} f^{\prime}(w) \frac{i w e^{-i t}}{1-w e^{-i t}}|\alpha(t)-C| d t+O\left(\lambda^{2}\right)\right) d w \\
& =z+\sum_{n=2}^{\infty}\left(a_{n}+\lambda \int_{t_{1}}^{t_{2}} \Delta a_{n}|\alpha(t)-C| d t\right) z^{n}+O\left(\lambda^{2}\right)
\end{aligned}
$$

where

$$
\delta a_{n}=\frac{2 i \cos \gamma}{n} \sum_{k=1}^{n-1}(n+k) k h_{k} e^{-(n-k) i t}
$$

and

$$
\Delta a_{n}=\frac{i}{n} \sum_{k=1}^{n-1} k a_{k} e^{-(n-k) i t}
$$

Thus, (we set $P_{0}(z)=\sum_{n=0}^{\infty} h_{n} z^{n}$ )

$$
\Phi\left(f_{*, a}\right)=\Phi(f)+\lambda \int_{t_{1}}^{t_{2}} \operatorname{Re}\left\{\sum_{j=2}^{n} d_{j} \delta a_{j}\right\}|\beta(t)-C| d t+O\left(\lambda^{2}\right)
$$

and

$$
\Phi\left(f_{*, b}\right)=\Phi(f)+\lambda \int_{t_{1}}^{t_{2}} \operatorname{Re}\left\{\sum_{j=2}^{n} d_{j} \Delta a_{j}\right\}|\alpha(t)-C| d t+O\left(\lambda^{2}\right)
$$

where $d_{j}=\left(\partial F / \partial X_{j}\right)\left(X_{2}, \ldots, X_{n}\right)$ at the extreme point $\left(a_{2}, \ldots, a_{n}\right)$. 
For $f(z)$ to be extremal for the problem under consideration it is necessary that the coefficient of $\lambda$ vanish in (6.2) and (6.3), i.e.,

$$
\int_{t_{1}}^{t_{2}} \operatorname{Re}\left\{\sum_{j=2}^{n} d_{j} \delta a_{j}\right\}|\beta(t)-C| d t=0
$$

and

$$
\int_{t_{1}}^{t_{2}} \operatorname{Re}\left\{\sum_{j=2}^{n} d_{j} \Delta a_{j}\right\}|\alpha(t)-C| d t=0 .
$$

But now, $Q_{1}(t)=\operatorname{Re} \sum_{j=2}^{n} d_{j} \delta a_{j}=0$ is equivalent to an equation of degree $2 n-2$ in $e^{i t}$ and therefore has at most $2 n-2$ roots with respect to $t$ for $-\pi \leqq t<\pi$. Similarly, $Q_{2}(t)=\operatorname{Re} \sum_{j=2}^{n} d_{j} \Delta a_{j}$ has at most $2 n-2$ zeros for $-\pi \leqq t<\pi$. Using Lemma 3.1 we conclude that $\alpha(t)$ and $\beta(t)$ are step functions each having at most $2 n-2$ jump points, and these points are the zeros of $Q_{1}(t)$ and $Q_{2}(t)$ respectively.

We now assume that $\alpha(t)$ and $\beta(t)$ each have $2 n-2$ jump points at $t_{1}, \ldots, t_{2 n-2}$ and $y_{1}, \ldots, y_{2 n-2}$ respectively. The variational functions $f_{* *, a}(z)$ and $f_{* *, b}(z)$ can now be constructed.

$$
\begin{aligned}
& f_{* *, a}(z)=z+\sum_{n=2}^{\infty}\left(a_{n}+\delta a_{n}\right) z^{n}, \\
& f_{* *, b}(z)=z+\sum_{n=2}^{\infty}\left(a_{n}+\Delta a_{n}\right) z^{n},
\end{aligned}
$$

where

$$
\delta a_{n}=\sum_{k=1}^{n-1} \frac{k h_{k}}{n}\left(e^{-(n-k) i y_{j}}-e^{-(n-k) i y_{j+1}}\right)
$$

and

$$
\Delta a_{n}=\sum_{k=1}^{n-1} \frac{k a_{k}}{n} \frac{e^{-(n-k) i t_{j}}-e^{-(n-k) i t_{j+1}}}{n-k}
$$

Computing $\Phi\left(f_{* *, a}\right)$ and $\Phi\left(f_{* *, b}\right)$ and exploiting the extremal property of $f(z)$ we arrive at the conditions

$$
\operatorname{Re} \sum_{k=2}^{n} d_{k} \delta a_{k}=0
$$

and

$$
\operatorname{Re} \sum_{k=2}^{n} d_{k} \Delta a_{k}=0
$$

Condition (6.4) implies that the function

$$
R(t)=\sum_{j=2}^{n} \operatorname{Re}\left(d_{j} \sum_{k=1}^{j-1} \frac{k h_{k}}{j} e^{-(j-k) t t}\right)
$$

has the same value at $y_{j}$ and $y_{j+1}$ and therefore its derivative with respect to $t$ must vanish at some point $y_{j}<t<y_{j+1}$. This implies that $Q_{1}(t)$ has more than 
$2 n-2$ zeros. Thus, we conclude that $\beta(t)$ hast at most $n-1$ jump points. A similar argument proves that $\alpha(t)$ can also have at most $n-1$ jump points.

Evaluating (6.1) with such $\alpha(t)$ and $\beta(t)$ we have the desired form.

Acknowledgement. The author wishes to express his gratitude to Professor Harry E. Rauch for his encouragement and many helpful suggestions during the course of research which led to this paper.

\section{REFERENCES}

1. L. Bieberbach, Aufstellung und Geweis des Drehungssatzes für schlichte konforme Abbildungen, Math. Z. 4 (1919), 295-305.

2. G. M. Goluzin, On a variational method in the theory of analytic functions, Leningrad. Gos. Univ. Ucen. Zap. 144 Ser. Mat. Nauk 23 (1952), 85-101; Amer. Math. Soc. Transl. (2) 18 (1961), 1-14.

3. M. Heins, Selected topics in the classical theory of functions of a complex variable, Holt, Rinehart and Winston, New York, 1962.

4. J. A. Hummel, A variational method for starlike functions, Proc. Amer. Math. Soc. 9 (1958), 82-87.

5. W. Kaplan, Close-to-convex schlicht functions, Michigan Math. J. 1 (1952), 169-185.

6. Z. Nehari, Conformal mapping, McGraw-Hill, New York, 1952.

7. S. Ogawa, A note on close-to-convex functions, J. Nara Gakugei Univ. 8 (1959), No. 2 , 9-10. (Quoted from Math. Rev. 31 (1966), 646, \#3588.)

8. M. S. Robertson, Variational methods for functions with positive real part, Trans. Amer. Math. Soc. 101 (1962), 82-93.

9. W. E. Kirwan, A note on extremal problems for certain classes of analytic functions, Proc. Amer. Math. Soc. 17 (1966), 1028-1031.

10. J. Kryz, Some remarks on close-to-convex functions, Bull. Acad. Pol. Sci. 12 (1964), $25-28$.

Yeshiva UNIVERSITY,

New York, New York 